\title{
INTERPRETIVE SUMMARIES, JANUARY 2010
}

Casein micelle dissociation in skim milk during high-pressure treatment: Effects of pressure, pH, and temperature. By Orlien et al., page 12. The pressure-induced dissociation of casein micelle in skim milk was found to be highly dependent on $\mathrm{pH}$ and less dependent on the temperature of the milk. This knowledge can be used in a targeted control of the production of HP-modified, functional micelle units useful for encapsulation, protection, and delivery of important nutrients in dairy products.

Neutral glycosphingolipid content of ovine milk. By Zancada et al., page 19. Milk glycosphingolipids have been reported to participate in the newborn's defense against pathogens. Ovine milk has a neutral glycosphingolipid pattern similar to human milk with a high content of globotriaosylceramide and globotetraosylceramide. These compounds could be used as an alternative and available source to supplement infant or bovine formulas with a view to preventing bacterial infection.

Effect of kefir grains on proteolysis of major milk proteins. By Ferreira et al., page 2\%. The proteolytic activity of kefir grains was evaluated by reverse phase-HPLC using pasteurized milk and cheese whey as substrate. The reduction of $\kappa^{-}, \alpha-$, and $\beta$-caseins, $\alpha$-lactalbumin $(\alpha-\mathrm{LA})$, and $\beta$-lactoglobulin $(\beta-\mathrm{LG})$ contents during 48 and $90 \mathrm{~h}$ incubation at $20^{\circ} \mathrm{C}$ was monitored. Significant proteolysis of $\alpha-\mathrm{LA}$ and $\kappa^{-}, \alpha-$, and $\beta$-caseins was observed. The effect of kefir amount was significant for $\alpha$-LA and $\alpha$ - and $\beta$-caseins. No significant reduction was observed with respect to $\beta$-LG concentration. Concerning the cheese whey-kefir samples, a similar behavior was observed for the $\alpha$-LA and $\beta$-LG proteolysis. Kefir grains are not appropriate as adjunct cultures to increase $\beta$-LG digestibility in whey-based or whey-containing foods.

Hot topic: Enhancing omega-3 fatty acids in milk fat of dairy cows by using stearidonic acidenriched soybean oil from genetically-modified soybeans. By Bernal-Santos et al., page 32. Very long chain omega-3 fatty acids such as eicosapentaenoic acid (EPA) are important for cardiac health and the prevention of chronic diseases, but food sources are limited. Stearidonic acid (SDA) is an omega-3 fatty acid that may be a surrogate for EPA because humans are able to convert it to EPA. Using SDA-enhanced soybean oil derived from genetically modified soybeans, we examined the potential to increase the omega- 3 fatty acid content of milk fat. We found that cows infused with SDA had higher milk fat concentrations of SDA and other omega-3 fatty acids derived from SDA including EPA. Thus, supplying SDA to dairy cows allows the production of dairy products that can provide omega-3 fatty acids in the diets of humans without changing fat intake.

Variability of mammary blood flow in lactating Holstein Friesian cows during the first twelve weeks of lactation. By Götze et al., page 38. Doppler sonography proved to be a reliable method for determining blood flow in the pudendoepigastric trunk of cows, with an intraindividual reproducibility of $96 \%$ and an interindividual reproducibility of $98 \%$. There was a high variability among cows, which was attributable to differences in the cross-sectional diameter of the vessel and blood flow velocity. The fluctuations in blood flow volume during lactation were caused by changes in blood flow velocity. Correlations between blood flow volume and actual milk yield in individual cows were moderate in this study.

Pressure distribution at the teat-liner and teatcalf interfaces. By van der Tol et al., page 45. During milking, vacuum and compression are applied to the cow's teats. It is assumed that vacuum and pressure concentrations tend to cause teat end injuries and hyperkeratosis. Although some liner designs show a reduction of teat loads, our results emphasize the unnatural nature of current milking technique compared with calf suckling. The analysis of teat pressure distribution at the teat-liner and the teat-calf interfaces shows promise and might be a first step in the development of a natural milking technique.

Biomechanics and histology of bovine claw suspensory tissue in early acute laminitis. $B y$ Danscher et al., page 53. Weakening of the tissues suspending the pedal bone in the claw capsule is the central issue in the theory of acute bovine laminitis. Nevertheless, clinical and histological signs of acute laminitis were not associated with decreased strength of claw suspensory tissue at the time points investigated. Tissue strength differed greatly between heifers originating from different herds and between different anatomical locations of the tissue samples. Knowledge of herd factors influencing claw tissue strength may be valuable in the prevention of claw lesions and lameness and the improvement of cattle welfare in intensive dairy production.

Suitability of low-dosage oxytocin treatment to induce milk ejection in dairy cows. By Belo and Bruckmaier, page 63. Chronic administration of high concentrations of oxytocin causes reduced spontaneous milk ejection in dairy cows. The aim of this study was to find a minimum oxytocin dosage that allows normal milk removal in cows with disturbed milk ejection 
while keeping the oxytocin plasma concentrations in a physiological range in order to avoid side effects of the oxytocin treatment. Milk flow duration in response to oxytocin injection increased with increasing oxytocin dosage. A desensitization of the udder toward oxytocin during both the actual milking and the chronic highdosage oxytocin treatment was observed. Nevertheless, low-dosage oxytocin treatments can minimize side effects.

Preferences of dairy cows for three stall surface materials with small amounts of bedding. $B y$ Norring et al., page 70. When only small amounts of straw bedding are provided, cows prefer to lay in stalls with soft rubber mat rather than stalls with concrete or sand floors. If dairy farmers need to reduce the amount of straw bedding used, rubber mats can help maintain cow comfort.

Optimal replacement policies for dairy cows based on daily yield measurements. By Nielsen et al., page 75. When to replace the cow during lactation from an economic point of view is an important problem in dairy industry. This paper describes the first step of developing a model for the dairy cow replacement problem that can be integrated into a modern herd management system. The model can be used to assist the farmer in replacement decisions on a daily basis and is based on daily milk yield measurements that are available in modern milking systems. Moreover, economic values are calculated for each cow based on its current state (lactation number, days in milk, production, and pregnancy status).

Susceptibility of Escherichia coli isolated from uteri of postpartum dairy cows to antibiotic and environmental bacteriophages. Part I: Isolation and lytic activity estimation of bacteriophages. By Bicalho et al., page 93. Puerperal metritis is associated with an abnormally enlarged uterus and a fetid, watery, red-brown uterine discharge, which are associated with signs of systemic illness. Escherichia coli and Arcanobacterium pyogenes are considered to be the most important bacteria associated with uterine infection. Bacteriophages are viruses that infect bacteria; they are obligate intracellular parasites and lack their own metabolism. In this study, several genetically diverse lytic bacteriophages with good antimicrobial activity against uterine $E$. coli isolates were successfully isolated from the manure lagoon of commercial dairy farms. The individual bacteriophage preparations had excellent lytic activity against their own host E. coli isolates.

Susceptibility of Escherichia coli isolated from uteri of postpartum dairy cows to antibiotic and environmental bacteriophages. Part II: In vitro antimicrobial activity evaluation of a bacteriophage cocktail and several antibiotics. By Santos et al., page 105. Metritis is one of the most important uterine disorders in cattle; it is caused by uterus contamination with bacteria. Escherichia coli is considered to be one of the most important bacterium involved in the establishment of metritis in cattle. Bacteriophage therapy, which uses virulent bacteriophages to control bacterial growth, has shown to be an attractive alternative to conventional drugs. We present in this study a phenotypic characterization of antimicrobial resistance in $E$. coli isolated from uterus fluid and the effect of bacteriophages in killing the isolates. The bacteriophage cocktail had a notable capacity to inhibit the in vitro growth of $E$. coli isolates and might be an attractive alternative to conventional treatment of metritis by reducing E. coli in uteri of postpartum dairy cows and consequently reducing the severity of clinical disease.

Costs and efficacy of management measures to improve udder health at Dutch dairy farms. By Huijps et al., page 115. Mastitis is a multifactorial disease, and there are a variety of management measures available to improve the mastitis situation at farm level. In this study, information about the effect of different management measures on bulk-tank somatic cell count and on the incidence of clinical mastitis for both environmental and contagious pathogens is given in relation to their associated costs. With this information available, farmers can make more informed decisions about mastitis management.

The amount of shade influences the behavior and physiology of dairy cattle. By Schütz et al., page 125. Few studies have examined how the amount of shade influences its use and benefits. Shade amounts of 2 sizes [large $\left(9.6 \mathrm{~m}^{2} / \mathrm{cow}\right)$ and small $\left(2.4 \mathrm{~m}^{2} / \mathrm{cow}\right)$ ] were evaluated and compared with no shade. Cows spent more time under the large shade, engaged in fewer aggressive interactions, and showed fewer signs of heat stress (e.g., they had lower respiration rates and spent less time around the water trough than cows with small shade or no shade). Cattle used shade to mitigate effects of heat load, but this behavioral strategy was effective only if adequate shade was provided.

Microbiological quality of raw milk utilized for small-scale artisan cheese production in Vermont: Effect of farm characteristics and practices. By D'Amico and Donnelly, page 134. United States Standards of Identity for cheese and cheese products permit the manufacture of more than 30 varieties of cheese from raw milk. This raises concerns because the presence of pathogenic bacteria in raw milk is well documented. This study fills gaps in data pertaining to the threat of emerging pathogens in raw milk intended for farmstead cheesemaking through the determination 
of overall quality and pathogen prevalence in raw milk destined for small-scale cheese manufacture. These data will help inform risk assessments that evaluate the microbiological safety of artisan cheeses, particularly those manufactured from raw milk.

Duration of weaning, starter intake, and weight gain of dairy calves fed large amounts of milk. $B y$ Sweeney et al., page 148. Abrupt weaning at 6 wk of age of calves fed large amounts of milk resulted in weight loss because the calves did not ingest sufficient starter before weaning. Gradual weaning over a $10-d$ period can stimulate starter intake and eliminate weight loss.

Nutrient availability and lactogenic hormones regulate mammary protein synthesis through the mammalian target of rapamycin signaling pathway. By Burgos et al., page 153. The mechanism by which protein synthesis in liver and muscle is stimulated following a meal is through nutrient- and hormone-mediated activation of an intracellular protein called mammalian target of rapamycin (mTOR). Here we show that amino acids are potent activators of mTOR and protein synthesis in bovine milk secretory cells. Lactogenic hormones had no effect on mTOR signaling by themselves, but activated mTOR and protein synthesis when amino acids or energy substrates were present. The results suggest that mTOR is a control point for mammary protein synthesis and should be studied to understand how nutrition of the dairy cow affects milk protein production.

The frequency of unilateral milking alters leucine metabolism and amino acid removal by the mammary gland of lactating goats. By Bequette and Douglass, page 162. One aspect of mammary metabolism lacking adequate detail is the metabolic requirement for amino acids. Several amino acids are extracted by the gland in excess of milk yield. The unilateral-milking model was used to alter milk synthesis by adjacent glands and test whether amino acid uptake and oxidation fluctuate with milk output. Leucine uptake and oxidation by the gland and blood flow were maintained or increased when milk synthesis was increased by frequent milking, suggesting that excess uptake of some amino acids is an obligatory requirement for milk synthesis.

Effects of heat stress and nutrition on lactating Holstein cows: II. Aspects of hepatic growth hormone responsiveness. By Rhoads et al., page 170. During heat stress, the magnitude of the decrease in milk production is beyond that which can be explained by reduced feed intake. Direct effects of heat stress on the somatotropic axis may be partially responsible for this discrepancy. Heat stress decreased hepatic growth hormone receptor abundance, phosphorylated signal transducer and activator of transcription (STAT)-5, and decreased IGF-I messenger ribonucleic acid abundance independent of reduced feed intake. Neither STAT5 protein abundance nor SOCS3 messenger ribonucleic acid concentrations changed during heat or nutritional stress. Knowledge gained during these experiments may facilitate development of new management strategies, which may alleviate the economic impact of heat stress on the dairy industry.

Identification of potential sources of Staphylococcus aureus in herds with mastitis problems. By Capurro et al., page 180. Staphylococcus aureus is a common bacterial cause of mastitis in dairy herds. Control programs are not always successful in preventing new infections, indicating that bacteria may be present in places other than those commonly considered. This study aimed at identifying sources of infection of importance for Staph. aureus mastitis in 5 Swedish dairy herds. Presence of Staph. aureus was investigated in milk from lactating cows and in body and environment samples of lactating cows, dry cows, late pregnant heifers, young heifers 4 to 12 mo old, and heifer calves 0 to 3 mo old. Identical Staph. aureus genotypes as in milk were found in a number of body and environmental sites. Among body sites, hock skin was the most important reservoir of Staph. aureus. The results can lead to improved strategies for udder health control.

Survival and replication of Mycoplasma species in recycled bedding sand and association with mastitis on dairy farms in Utah. By Justice-Allen et al., page 192. Mycoplasma spp. cause infectious bovine mastitis, and transmission occurs through milking equipment and direct animal contact. Mycoplasma bovis was discovered in high numbers in recycled bedding sand at a dairy experiencing an outbreak of clinical mycoplasma mastitis. Mycoplasma spp. survived in sand for $8 \mathrm{mo}$ and its concentration in sand over time was associated with changes in temperature, precipitation, and the concentration of other microorganisms. Recycled bedding sand appears to be a possible environmental source of Mycoplasma spp. that can infect dairy cows. Results warrant further investigation of the role of this environmental source in the epidemiology of mycoplasma mastitis.

Continuous lactation effects on mammary remodeling during late gestation and lactation in dairy goats. By Safayi et al., page 203. Omitting the dry period is possible in goats. Although continuous lactation suppressed mammary epithelial cell (MEC) renewal because of suppression of prepartum cell proliferation, the rate of apoptosis was unaffected. Studies on MEC gene expression could not account for this. Continuous lactation glands throughout lactation had a larger microvessel proportion and a lower proportion 
of MEC that were more fully differentiated compared with normal lactating glands. If fully differentiated MEC are older and have more secretory activity, this may in part explain why goats can lactate continuously without major negative effect on overall milk yield.

Therapeutic effects of antimicrobial treatment during lactation of recently acquired bovine subclinical mastitis: Two linked randomized field trials. By van den Borne et al., page 218. Two linked randomized field trials were performed to determine therapeutic effects of antimicrobial treatment of recently acquired subclinical mastitis during lactation. Treated quarters had a higher probability of bacteriological cure than control quarters for all pathogens in both trials. Treatment resulted in lower quarter and composite somatic cell count, whereas milk yield was not affected by treatment. Pathogen species, quarter somatic cell count, and infectious status before intervention were related to bacteriological cure of recently acquired subclinical mastitis. Cow-level factors were not related to bacteriological cure. Subclinical Staphylococcus aureus mastitis tended to have a higher cure after early treatment.

Prediction of the herd somatic cell count of the following month using a linear mixed effect model. By Lievaart et al., page 234. A short-term prediction of average herd somatic cell count would be a useful additional tool to support udder health management decisions. A linear mixed effect model that included somatic cell count data, herd characteristics, seasonal fluctuations, and management practices was used to predict the herd-average somatic cell count of the next month. The model accurately predicted $81 \%$ of the herd somatic cell count for the following month within a range of 30,000 cells $/ \mathrm{mL}$ compared with $59 \%$ in a model without explanatory variables. This model could be a useful addition to dairy herd health programs using Dairy Herd Improvement data.

Forced heat loss from body surface reduces heat flow to body surface. By Berman, page 242. In humid climates, heat removal from the body surface by coat wetting and forced air-flow cycles is a powerful means for heat stress relief. Heat removal from the body core depends upon heat flow to body surface. Half-body surface temperature was monitored in 4 cows during successive cooling cycles. Cooling for 35 min reduced the temperature of the body shell but not the body core. Cooling for $1 \mathrm{~h}$ or more reduced core temperature, suggesting that initial cooling of the body shell is followed by subsequent heat transfer from the body core to shell. Evaluation of cooling duration is needed for optimization of heat stress relief.
On the use of physical activity monitoring for estrus detection in dairy cows. By Løvendahl and Chagunda, page 249. Improvement of fertility in dairy cows is needed in order to balance side effects of selection for higher milk yield. However, fertility measures based on artificial insemination data are biased by farm management. Electronic behavior sensors were used for detection of estrus and were shown to give important information for fertility traits and to be free of bias from management decisions.

Short communication: Transport of 2-hydroxy4-methyl-thio-butanoic isopropyl ester by rumen epithelium in vitro. By Breves et al., page 260. This study evaluated the ability of rumen epithelium to absorb an ester of methionine hydroxy analog, a proposed methionine supplement for ruminants, which has been postulated as being absorbed directly from the rumen. However, rumen epithelium exhibited little ability to transport this analog. There was transport of a breakdown product, methionine hydroxyl analog, but it was not sufficient to explain a rapid absorption. This indicates that previously reported rapid increases in blood methionine as a result of feeding methionine hydroxy analog are not caused by direct absorption from the rumen but must arise by another mechanism.

Shifts in bacterial community composition in the rumen of lactating dairy cows under milk fatdepressing conditions. By Weimer et al., page 265. Milk fat depression (MFD) reduces dairy profitability and is thought to be caused in part by the activities of rumen bacteria. We identified cows that displayed MFD when fed a diet that contained rapidly fermented starch, with or without the feed additive monensin, and showed that the bacterial populations shifted when the cows went into MFD. One specific bacterial type became much more abundant in most, but not all, cows that displayed MFD. This bacterial type may be an important contributor to MFD in some cows.

pH dynamics and bacterial community composition in the rumen of lactating dairy cows. $B y$ Palmonari et al., page 279. The degree of acidity in the rumen is known to affect certain microbial species, but its effect on the whole rumen bacterial community is largely unknown. Using methods that analyze both cultured and not-yet cultured bacteria, we found that there is little relationship between rumen acidity and the structure of the rumen bacterial community. Some cows that displayed normal acidity profiles had unique rumen bacterial communities and also produced milk with low fat content. The low fat test was associated with elevated levels of one bacterial type and reduced levels of another. 
Lactation performance and amino acid utilization of cows fed increasing amounts of reducedfat dried distillers grains with solubles. By Mjoun et al., page 288. Reduced-fat dried distillers grains with solubles (RFDG; $3.5 \%$ crude fat) were fed up to $30 \%$ on a dry matter basis to mid-lactation Holstein cows. Feeding RFDG had no effect on milk yield and feed intake. Milk fat percentage and yield, fat-corrected milk, and total solids in milk were increased at 30\% RFDG. Feed efficiency tended to increase as RFDG increased in the diet. Milk protein percentage was maximized at $20 \%$ inclusion of RFDG.

Association between bulk-tank milk Salmonella antibody level and high calf mortality in Danish dairy herds. By Nielsen et al., page 304. Calf mortality in Denmark is considered to be too high and the Danish Cattle Federation initiated a campaign in 2008 to reduce it. We used registry data to investigate associations between risk factors and mortality for calves from 1 to $180 \mathrm{~d}$ of age. High calf mortality in dairy herds was associated with high Salmonella bulk-tank milk antibody level, organic production, and purchase of animals, and mortality was higher for the Jersey breed than for large breeds. This study showed that controlling or eradicating Salmonella might aid in reducing calf mortality in the dairy sector.

Carbohydrate source and protein degradability alter lactation, ruminal, and blood measures. By Hall et al., page 311. Do dairy cows perform differently when fed combinations of feeds that differ in carbohydrate type or rumen protein degradability? We found that cows fed corn grain (a starch source) produced more milk and were more efficient when more of their dietary protein was rumen-degradable, whereas cows fed citrus pulp (pectin + sugars) or molasses and sucrose (sugars) were more efficient and made more milk when fed more rumen-undegradable protein. We can use the knowledge that feed carbohydrate source and protein characteristics affect production to formulate diets that more closely meet animals' needs to keep them healthy, productive, and environmentally friendly.

Effective nitrogen preservation during urine collection from Holstein heifers fed diets with high or low protein content. By Knowlton et al., page 323. Acidification of urine upon collection is common practice to manage potential losses of nitrogen before analysis. This methodology is adequate for assessment of nitrogen utilization by livestock, but is not conducive to research focused on postexcretion changes in manure nitrogen. We compared nitrogen preservation in urine acidified at the start of collection, acidified after $6 \mathrm{~h}$ collection, or chilled immediately. Regardless of dietary protein content, the 3 collection methods were equally effective in preserving nitrogen during urine collection.
The chilled immediately approach may be useful for studies focused on ammonia emission.

Genetic determination of fatty acid composition in Spanish Churra sheep milk. By Sánchez et al., page 330. Under current production conditions, not enough additive genetic variation is involved in the composition of fatty acid contents in Churra sheep milk fat. Thus, unlike dairy cattle, implementing a selective breeding program relying on evaluation based on the infinitesimal additive genetic model does not seem to be an appropriate management tool for modifying the fatty acid profile in the milk of this breed.

Polymorphisms in the bovine leptin gene associated with perinatal mortality in Holstein-Friesian heifers. By Brickell et al., page 340. Leptin has a functional role during pregnancy, signaling nutritional status between the mother and fetus. Dairy heifers suffer high rates of perinatal mortality (stillbirths and mortality of calves within $24 \mathrm{~h}$ of parturition) at first calving. Single nucleotide polymorphisms (variation in the base sequence occurring at any given single position in a gene) in the leptin gene were associated with differences in the level of perinatal mortality. The potential exists to use this genetic information as a tool to aid in selection, with the aim of reducing perinatal mortality.

Detection of QTL affecting the milk fatty acid profile in sheep chromosome 22: Role of the stearoyl-CoA desaturase gene in Spanish Churra sheep. By García-Fernández et al., page 348. Most of the conjugated linoleic acid in ruminant milk is synthesized in the mammary gland by the action of the enzyme stearoyl-CoA desaturase (SCD) on circulating vaccenic acid. We have analyzed a commercial population of Spanish Churra sheep for the detection of genes on ovine chromosome 22 , location of the SCD gene that influences milk fatty acid composition, where the SCD gene has been mapped. Specifically, the relationship between allelic variants of the SCD gene and the ratio of conjugated linoleic acid:vaccenic acid was studied.

Economic values and expected effect of selection index for pathogen-specific mastitis under Danish conditions. By Sørensen et al., page 358. Economic costs related to pathogen-specific mastitis were estimated using a stochastic simulation model to be between $€ 149$ and $€ 570$ per case. In comparison, the cost of a mastitis case with unknown pathogen was $€ 231$. The costs were highest for contagious pathogens such as Staphylococcus aureus and coagulase-negative staphylococci. Genetic superiorities of an index of several pathogen-specific mastitis traits and an index containing unspecific mastitis were equally good. Inclusion of somatic cell count information in the indices had the highest effect on selection differentials when pathogen- 
specific mastitis traits were considered because of lower heritability of the latter.

Short communication: Genetic and environmental relationships between milk yield and kidding interval in dairy goats. By Montaldo et al., page 370. Genetic and environmental parameters for first-parity milk yield and interval between first and second kidding were determined for Alpine, LaMancha, Nubian, Saanen, and Toggenburg dairy goats. Across-breed heritability was $36 \%$ for first-parity milk yield and $5 \%$ for first kidding interval. Increased milk yield was associated with longer kidding intervals both genetically and environmentally. Fertility should be included as a selection criterion in dairy goat breeding programs to avoid its decline.

Conceptual modeling of postmortem evaluation findings to describe dairy cow deaths. $B y$ McConnel et al., page 373. Dairy cow mortality levels in the United States are excessive and increasing over time. Combating rising mortality is dependent upon more clearly defining the reasons that cows die through thorough necropsy-based postmortem evaluations. Although postmortem evaluations provide the relevant information necessary for framing a cow's death, a restructuring of on-farm databases will be required if this level of detail is to be integrated into useful monitoring systems. Without database development that can capture this information for future evaluation, it cannot be used to guide management.

Determinants of technical efficiency among dairy farms in Wisconsin. By Cabrera et al., page 38\%. Data from 273 Wisconsin dairy farms were used to examine the extent to which technical efficiency is related to practices commonly used by dairy farmers and the effect of intensification on the performance of the farms. The empirical analysis showed that, at a commercial level, the administration of bovine somatotropin hormone to lactating cows increases milk production. In addition, farm efficiency is positively related to farm intensification, the level of contribution of family labor in the farm activities, the use of a total mixed ration feeding system, and the milking frequency.

A large Markovian linear program for replacement policies to optimize dairy herd net income for diets and nitrogen excretion. By Cabrera, page 394. A novel bioeconomic optimization model for dairy herd dynamics was developed to study the effect of replacement policies that optimize dairy herd net income and nitrogen excretion. The model was used to study 5 diets. High-concentrate diets maximized net revenue and high-forage diets minimized $\mathrm{N}$ excretion. The optimal cow replacement policy varied for each scenario, but in general, the model always suggested to 1) keep pregnant cows, 2) keep primiparous cows longer than multiparous cows, and 3) decrease replacement rates when income over feed cost is high. Implementation of the model is an important advancement in dairy decision making.

Manure nutrient excretion by Jersey and Holstein cows. By Knowlton et al., page 40\%. Manure nutrient excretion by sixteen dairy cows $(8$ Holstein and 8 Jersey) was evaluated to quantify differences caused by breed. Total manure excretion was lower in Jersey cows than in Holstein cows, with reductions generally proportional to changes in feed intake. The American Society of Agricultural and Biological Engineers tabular values for daily manure excretion derived from Holstein cows are not appropriate for Jersey cows, but the manure solids prediction equations that include animal and dietary factors may be appropriately applied to Jersey cows. Accounting for breed differences in manure excretion will support more effective nutrient management planning on dairy farms.

Optimizing weaning strategies of dairy replacement calves. By Bach et al., page 413. Weaning is a stressful period for calves and it is typically characterized by decreased animal performance and, on occasion, increased incidence of respiratory afflictions. Common field recommendations advocate for keeping calves individually housed for at least 1 or 2 wk following weaning. This study demonstrates that moving calves into groups immediately after weaning is more desirable than keeping calves individually housed for additional days. Furthermore, this study also shows that moving calves to groups at $49 \mathrm{~d}$ of life and weaning them while in groups results in greater solid feed intakes, calf performance, and improved health.

Effect of synchronization and semen sorting on artificial insemination bull fertility. By Abdel-Azim, page 420. Data were collected from 438 commercial dairy herds over a period of $2 \mathrm{yr}$ for the purpose of evaluating artificial insemination bulls for their fertility with synchronization protocols and semen sorting. The study showed that bull rankings, produced after accounting for whether the insemination was administered on a synchronized female or not, differed from rankings that ignored this information. The study also showed that bulls differed significantly for their rankings based on conventional and sex-sorted semen. Bull fertility evaluation models should account for synchronization and semen sorting. 